\title{
Correlation of Fibromyalgia Survey Questionnaire and Quantitative Sensory Testing Among Patients With Active Rheumatoid Arthritis
}

Meriah N. Moore ( $\nabla$ momeriah@umich.edu )

University of Michigan https://orcid.org/0000-0003-4502-9993

Beth I. Wallace

University of Michigan

Jing Song

Northwestern University Feinberg School of Medicine

Lutfiyya N. Muhammad

Northwestern University Feinberg School of Medicine

Andrew C. Heisler

Northwestern University Feinberg School of Medicine

Daniel J. Clauw

University of Michigan

Mary B. Bolster

Massachusetts General Hospital

Wendy Marder

University of Michigan

Tuhina Neogi

Boston University School of Medicine

Alyssa Wohlfahrt

Tufts University School of Medicine

Dorothy D. Dunlop

Northwestern University Feinberg School of Medicine

Yvonne C. Lee

Northwestern University Feinberg School of Medicine

Research article

Keywords: Pain centralization, fibromyalgia, rheumatoid arthritis, fibromyalgia survey questionnaire, quantitative sensory testing

Posted Date: July 20th, 2021 
DOl: https://doi.org/10.21203/rs.3.rs-715260/v1

License: (c) (1) This work is licensed under a Creative Commons Attribution 4.0 International License. Read Full License 


\section{Abstract}

\section{Background}

Patients with rheumatoid arthritis (RA) commonly demonstrate disordered pain processing, termed pain centralization. Heightened pain sensitivity and pain pathways associated with pain centralization are often assessed using quantitative sensory testing (QST), which is not routinely available and burdensome to patients. The self-administered fibromyalgia survey questionnaire (FSQ) has been proposed as a low-burden, surrogate measure of pain centralization. We examined the correlation between FSQ and QST as measures of pain centralization in a cohort of patients with active RA.

\section{Methods}

Participants in the multicenter Central Pain in Rheumatoid Arthritis (CPIRA) cohort underwent FSQ and QST evaluation at enrollment. QST measures included pressure pain threshold (PPT) at the thumb, trapezius, wrist and knee; temporal summation (TS) at the wrist and arm; and conditioned pain modulation (CPM). Partial Spearman correlation was assessed between FSQ and each QST measure, adjusted for age, sex, race, body mass index, serologic status, swollen joint count, C-reactive protein, pain catastrophizing, and study site. We performed sensitivity analyses including a) stratified analysis by sex and b) correlations between the two sub-components of the FSQ (widespread pain index [WPI], and symptom severity scale [SSS]) and QST measures.

\section{Results}

Among 285 participants with high RA disease activity (mean baseline Clinical Disease Activity Index score 24.56), FSQ was weakly but statistically significantly correlated with PPT ( $r=-0.21$ to -0.31 ) and TS ( $r=0.13$ to 0.15$)$ at all sites in unadjusted analyses. After adjustment, statistically significant correlations persisted for PPT at all sites except the thumb, and for TS at the wrist Sensitivity analyses did not identify differences in association based on sex or with the components of the FSQ (WPI or SSS).

\section{Conclusions}

FSQ and QST were correlated among participants with active RA, but the strength of association was modest. This may be due to the high level of RA disease activity of the cohort studied. Both QST and the FSQ are likely imperfect singular measures of pain centralization.

\section{Background}

Patients with rheumatoid arthritis (RA) frequently have abnormalities in pain processing, which are characterized by aberrant functioning of nociceptive neural pathways, leading to somatic hypersensitivity. ${ }^{1}$ This process is termed pain centralization, and the pain associated with this process is called centralized pain or nociplastic pain. ${ }^{2}$ Pain centralization is associated with worse functional outcomes and reduced response to disease-modifying treatment. ${ }^{3-5}$ In the research context, pain 
centralization is often assessed by quantitative sensory testing (QST), which uses established protocols to measure the detection threshold of standardized noxious stimuli. ${ }^{6}$ QST has been previously used to characterize pain centralization in RA, ${ }^{1,7,8}$ but QST poses a substantial burden to patients as it is timeconsuming and requires a trained operator to administer the tests in a controlled setting.

The self-administered fibromyalgia survey questionnaire (FSQ) has been proposed as a low-burden surrogate to QST assessment. $9,10,11$ The FSQ assesses widespread pain and somatic symptoms like fatigue, poor sleep and cognitive difficulty. ${ }^{12}$ However, there are limited data evaluating the relationship between the clinical symptoms measured using FSQ and the physiologic abnormalities measured by QST. Previous studies in non-inflammatory pain conditions, and in patients with well-controlled RA, suggest a low-moderate correlation between these measures $(|r|=0.27-0.44)$, which may be limited to certain subpopulations (i.e. female patients). ${ }^{9,13,14}$ Further, widespread pain and somatic symptoms may be driven by other processes beyond pain centralization, and therefore may reflect distinct domains contributing to the pain experience in RA.

To our knowledge, no data exist regarding the relationship between pain centralization assessed by QST vs. FSQ among patients with active RA. The assessment of pain centralization is particularly important in this subgroup because pain centralization may inflate composite disease activity measures, making it seem as if these patients have active inflammatory disease when they do not. Identification of pain centralization in these patients could impact treatment decisions about escalating disease modifying anti-rheumatic drug (DMARD) therapy and may inform alternative management approaches to target pain centralization. To address this gap in knowledge, we aim to examine the correlation between FSQ and QST in a cohort of participants who require intensification of DMARD treatment for active RA.

\section{Methods}

\section{Study_population}

Central Pain in Rheumatoid Arthritis (CPIRA) is comprised by participants enrolled prospectively with active RA who are changing DMARD therapy due to uncontrolled disease activity. ${ }^{1}$ Between January 2014 and July 2017, 295 participants at five U.S. academic medical centers enrolled in CPIRA. Exclusion criteria included: a) failure to meet 2010 American College of Rheumatology/European League Against Rheumatism criteria for RA diagnosis; b) a coexisting diagnosis of any other systemic autoimmune disease, severe Raynaud phenomenon, peripheral vascular disease, or peripheral neuropathy, and c) use of chronic opiates, changing dose of centrally acting pain medications in the past three months, or prednisone $\geq 10 \mathrm{mg} /$ day. Written informed consent was provided by all participants. The institutional review board at each site approved the study.

We used baseline data from CPIRA for this study. Analyses were restricted to 285 participants with data in at least one of the seven QST measures as well as complete data in FSQ and covariates. Ten participants were excluded due to missing data in covariates (i.e. race or C-reactive protein [CRP]). 
Variables including age, sex, RA disease duration, RA serostatus, body mass index (BMI), and enrollment site were assessed at the baseline study visit. Height and weight were measured to calculate BMI [weight $(\mathrm{kg})] /\left[\right.$ height $\left.\left(\mathrm{m}^{2}\right)\right]$. Presence of rheumatoid factor $(>14 \mathrm{lU} / \mathrm{ml})$ and cyclic citrullinated peptide antibody $(>17 \mathrm{U})$ was assessed through serum analysis, performed at a central laboratory. Patient-reported questionnaires provided demographic and RA disease duration information.

\section{Assessment of RA disease activity and inflammation}

RA disease activity was assessed through measurement of CRP and calculation of the Clinical Disease Activity Index (CDAl) which includes tender joint count (TJC), swollen joint count (SJC), patient global assessment and assessor global assessment. ${ }^{15,16}$ Trained study staff members performed standard 28joint counts and assessor global assessments. Responses for patient global assessment were measured on a 100-point scale.

\section{Assessment of pain centralization}

\section{Quantitative sensory testing (QST)}

We evaluated three baseline QST measures: pressure pain threshold (PPT), temporal summation (TS), and conditioned pain modulation (CPM). We performed interrater reliability assessments across four assessors for both PPT and TS; intraclass correlation coefficients for both measures ranged from 0.71 to 0.90 , which is considered good to excellent. ${ }^{17}$

PPT, which assesses hyperalgesia, was measured using a Wagner Force 10 FDX algometer with a $1 \mathrm{~cm}^{2}$ probe placed at the bilateral trapezius muscles, wrists, knees and thumbnails. PPTs assess overall sensitivity to pain. Low PPTs at joint sites only indicate peripheral mechanisms of sensitization, whereas low PPTs at both joint and non-joint sites indicate pain centralization. Pressure was increased by 0.5 kilopounds force (kpf) per second until the participant reported pain at each assessment site. PPT was defined as the pressure at which the participant reported pain with lower values suggesting more sensitivity.

TS assesses amplification of painful inputs in response to repeated stimuli and is considered a specific measure of pain facilitation, termed central sensitization. We measured TS using 6 weighted probes (8 256 millinewton $(\mathrm{mN})$ ) placed on the participant's wrist and forearm. Probe weight was increased until the participant reported a pain score of $30-40 / 100$, or the heaviest weight was reached. The probe registering a $30-40 / 100$ pain score was then tapped against the wrist and dorsal forearm 10 times, with 0.5 seconds for each tap and 1 second between taps. After taps 1, 5, and 10, the participant rated pain on a $0-100$ scale. We subtracted the participant's pain score at tap 1 from the score at tap 10 , then divided by 10 to provide a TS score from $0-10$. Higher TS scores represent higher pain amplification. 
CPM is believed to be a measure of descending inhibitory pain modulation. The conditioning stimulus engages the descending (inhibitory) analgesic pathway, while the second stimulus tests the effect of this inhibition. In an appropriately functioning pathway, the inhibition results in a lessened pain response to the second stimulus. Our conditioning stimulus was a cold water bath at $5-7 \mathrm{C}$, into which participants placed their right hand. We assessed PPT at the left trapezius muscle at two time points: before the cold water bath, and 20 seconds after initiation of the cold water bath. CPM was reported as the ratio of PPT at the second time point to PPT at the first time point, multiplied by 100 , with lower values suggesting inefficient descending analgesic inhibition.

\section{Assessment of fibromyalgia severity}

All participants completed the 2016 revised version of the 2010/2011 Fibromyalgia Survey Questionnaire (FSQ) at baseline. ${ }^{18}$ This instrument is composed of a widespread pain index (WPI) which assesses selfreported pain at 19 pre-specified sites, and a $0-12$ symptom severity scale (SSS). SSS measures the sum of self-reported fatigue, nonrestorative sleep, and cognitive symptoms on a $1-3$ point Likert scale and the presence of headache, abdominal pain, and depression assessed as binary variables. This questionnaire has been previously used to measure severity of fibromyalgia, the prototypical centralized pain condition, in the general population as well as in disease-specific cohorts, including the CPIRA cohort. ${ }^{1,19}$

\section{$\underline{\text { Statistical analysis }}$}

Descriptive statistics were used to evaluate demographic and clinical data. The primary analysis evaluated the Spearman correlation between each QST measure (PPT, TS, CPM) and overall FSQ score. Partial correlation adjusted for age, sex, race, BMI, study site, seropositivity, CRP, SJC, and pain catastrophizing (assessed by the Pain Catastrophizing Scale) ${ }^{20}$ We performed a sex-stratified sensitivity analysis to examine the possibility suggested from literature that sex may modify the correlation between FSQ and QST measures. ${ }^{9}$ A second sensitivity analysis evaluated the correlation between QST and each FSQ component: WPI which assesses the extent of pain, and SSS which assesses the severity of comorbid non-pain symptoms.

\section{Results}

We describe the characteristics of the 285 participants included in this study in Table 1. Mean age was 54.7 (standard deviation (SD) 13.7) years, $82 \%$ were female, $75 \%$ were Caucasian, and $78 \%$ were seropositive. Mean (SD) baseline CDAl score was 24.56 (14.25), representing high RA disease activity. ${ }^{16}$ Mean (SD) baseline FSQ score was 11.22 (6.08) out of 31 total possible with $38 \%$ of the study population meeting diagnostic criteria for fibromyalgia.

In unadjusted analyses, FSQ had a statistically significant, but weak inverse correlation between FSQ and PPT at all sites, including the thumb ( $=-0.21$ (95\% confidence interval $[\mathrm{Cl}]-0.32,-0.10)$ ), trapezius $(=$ 
$-0.25(95 \% \mathrm{Cl}-0.35,-0.13)$ ), wrist $(=-0.27(95 \% \mathrm{Cl}-0.37,-0.16))$, and knee $(=-0.31(95 \% \mathrm{Cl}-0.41,-0.20)$ Weak correlations were also found between FSQ and TS at the wrist $(=0.15(95 \% \mathrm{Cl} 0.03,0.26))$, and arm ( $=0.13(95 \% \mathrm{Cl} 0.01,0.24))$. Adjusting for covariates reduced the magnitude of these correlations, but correlations between FSQ and PPT at the trapezius ( $=-0.13(95 \% \mathrm{Cl}-0.25,-0.01))$, wrist $(=-0.16$ $(95 \% \mathrm{Cl}-0.27,-0.04))$, and knee $(=-0.20(95 \% \mathrm{Cl}-0.32,-0.09))$, as well as TS at the wrist $(=0.13(95 \% \mathrm{Cl}$ $0.01,0.24)$ ) remained statistically significant. No significant correlation was found between FSQ and CPM (Figure 1).

To examine the previously reported effect of sex on the relationship between FSQ and QST, we examined Spearman correlations of FSQ and QST by sex. ${ }^{9}$ Individually, correlations for men and women were similar in magnitude and statistical significance to the overall analysis. The largest difference occurred in the correlations of FSQ with PPT of the trapezius, but no meaningful pattern related to sex was observed (Table 2).

To evaluate for differences in the strength of relationship between QST and each component of the FSQ, we examined how each QST measure correlated with WPI and SSS (Table 3). For PPT, the magnitude of the observed correlations for SSS (range $=-0.25$ to -0.31 ) was similar to those seen in the primary analysis, while those for WPI were lower than those seen in the primary analysis (range $=-0.13$ to -0.23 ). Weak correlations were found between SSS and TS of wrist ( $=0.16(95 \% \mathrm{Cl} 0.04,0.27))$ and TS of the arm ( $=0.13(95 \% \mathrm{Cl} 0.01,0.24))$ while no correlations were found between WPI and TS. No correlations were found with either FSQ components and CPM.

\section{Discussion}

In a cohort of patients with RA requiring escalation of DMARD therapy due to uncontrolled disease activity, we found that FSQ was weakly correlated with PPT and TS, and not correlated with CPM. These relationships did not differ by sex when compared to the overall analysis. In a sensitivity analysis, the correlations between both components of FSQ (SSS and WPI) and QST measures were minimally different. These results indicate that, among patients with active RA, the patient-reported symptoms measured by FSQ are not strongly associated with quantitative measurements of pain centralization assessed by QST. Thus, while the FSQ may reflect severity of FM in terms of symptoms, it may not provide additional insights into altered nociceptive signal processing.

The relationship between patient-reported outcome measures like FSQ, and quantitative assessments like QST, may be influenced by a patient's underlying disease state and associated type of pain pathology. Prior work has shown moderate correlations between PPT and self-reported pain measures (i.e. McGill Pain Questionnaire) among patients with non-inflammatory conditions like fibromyalgia and chronic fatigue syndrome. ${ }^{21,22}$ In contrast, reported widespread pain was independent of PPT in a study of patients with knee osteoarthritis. ${ }^{23}$ Our work shows that patients with active RA, a highly inflammatory condition, demonstrate weak correlations between FSQ and QST. One explanation for this finding may be that the FSQ, in addition to detecting widespread muscle pain typical of pain centralization, is capturing 
inflammatory joint pain in patients with active RA. This explanation is supported by our group's previous unpublished finding that swollen joint count and CRP increase with increasing FSQ.

In our secondary analysis, we did not see a change in the correlation of QST and FSQ when stratified by sex. This is in contrast to a prior study of patients with knee osteoarthritis, where there was a strong correlation of FSQ and PPT among female patients, but no correlation among male patients. ${ }^{9}$ The authors hypothesized that this finding may be related to sex differences in pain characteristics because females in their study had higher FSQ scores, higher pain hypersensitivity measured by PPT, as well as higher rates of depression, anxiety, and pain catastrophizing. While our analysis is limited by the small percentage of men $(17.8 \%)$, prior work has revealed mixed results regarding the influence of sex on experimental pain models. ${ }^{24}$ It is also possible that the role of sex as a modifier of the relationship between FSQ and QST depends on other factors, such as disease type (e.g., osteoarthritis vs. RA).

We also considered the hypothesis that the separate components of the FSQ may be differentially associated with QST measures. The SSS-component of the FSQ assess symptoms (fatigue, waking unrefreshed, cognitive symptoms, headaches, lower abdominal pain, depression), which are a part of the syndrome of fibromyalgia but may not be directly related to pain sensitivity and may also be due to other causes. In contrast, the WPI-component of the FSQ focuses specifically on pain distribution. Thus, we performed a sensitivity analysis to separately examine associations between QST and the two subcomponents of the FSQ (WPI and SSS). However, the strength of the correlations between QST and each component of the FSQ were not meaningfully different (Table 3).

Our study has notable strengths. To our knowledge, this is the first study to evaluate the relationship between examiner-derived and patient-reported measurements of pain centralization in patients with an active inflammatory condition. Patients with co-existing inflammatory pain have historically been understudied in pain research, ${ }^{25}$ despite the high prevalence and well-documented morbidity caused by disorders of pain centralization in this population. ${ }^{26,27}$ This study uses data from CPIRA, one of the only cohorts to systematically collect examiner-derived and patient-reported pain measures in a population with a systemic inflammatory condition.

There are several limitations to our work. The study is cross-sectional, and causation cannot be determined from these observational data. Although QST is commonly used to assess pain sensitivity and, thereby, yield inferences about peripheral and central pain pathways, there is no gold standard for assessing pain centralization. Prior work has questioned the use of QST as a reference standard. For example, in patients with low back pain, QST had limited prognostic value for predicting the development of chronic symptoms or treatment failure after surgery. ${ }^{28,29}$ Both QST and FSQ measures typically correlate only modestly with functional neuroimaging techniques that are considered by some experts to be superior to either measure. ${ }^{30,31}$ These results do not mean that FSQ or QST do not provide useful information, only that the two measures are capturing different concepts. Finally, the goal of this study was to assess the correlation between FSQ and QST-assessed pain centralization in patients with active RA. Thus, our results do not necessarily extend to patients with well controlled inflammatory arthritis. 


\section{Conclusions}

In conclusion, these results do not support the use of FSQ as a proxy measurement for QST among patients with active RA. The difference between our results and results from non-inflammatory pain conditions suggests that population-specific characteristics likely impact the performance of these measures. While FSQ and QST each provide valuable information, they do not appear to assess the same construct in this population with high levels of inflammatory pain.

\section{List Of Abbreviations}

Rheumatoid arthritis (RA)

Fibromyalgia Survey Questionnaire (FSQ)

Quantitative sensory testing (QST)

Central Pain in Rheumatoid Arthritis (CPIRA)

Pressure pain threshold (PPT)

Temporal summation (TS)

Conditioned pain modulation (CPM)

Widespread pain index (WPI)

Symptom severity scale (SSS)

Disease modifying anti-rheumatic drug (DMARD)

C-reactive protein (CRP)

Body mass index (BMI)

Clinical Disease Activity Index (CDAl)

Tender joint count (TJC)

Swollen joint count (SJC)

Standard deviation (SD)

Confidence interval (Cl)

Massachusetts General Hospital (MGH) 


\section{Declarations}

\section{Ethics approval and consent to participate}

Written informed consent was provided by all patients. Each site's institutional review boards approved the study.

\section{Consent for publication}

Not applicable

\section{Availability of data and materials}

The dataset supporting the conclusions of this article are not publicly available due to dataset containing information that could compromise research participant privacy and consent.

\section{Competing interests}

BW is supported by the National Institutes of Health 5KL2TR002241-04

YL has received consulting fees, speaking fees, and/or honoraria from Eli Lilly (less than $\$ 10,000$ each), research support from Pfizer, and stock ownership in Cigna.

MB has received consulting fees and/or honoraria from Gilead Sciences $(<\$ 10,000)$ and stock ownership in Johnson and Johnson.

DC has received consultant fees from Pfizer Inc $(>\$ 10,000)$ and Aptinyx, Daiichi Sankyo, Eli Lilly, Intec Pharma, Samumed, Theravance, Tonix, Zynerba $(<\$ 10,000)$, and expert testimony for Eli Lily, Pfizer Inc, Nix Patterson LLP, Williams \& Connolly LLP.

\section{Funding}

The CPIRA study was funded by NIH/NIAMS R01 AR064850. Drs. Lee and Muhammad and Ms. Song were also supported by NIH/NIAMS P30 AR072579. Beth I. Wallace, MD, MSc was supported by NIH/NCATS 5KL2TR002241-04. Dr. Neogi was supported by NIH P30 AR072571 and K24 AR070892.

\section{Authors' contributions}

MM and BW: Conceptualization, study design/methodology, data interpretation, writing-original draft. MM and BW contributed equally.

AH: Data interpretation, writing-review and editing

JS and LM: Study design/methodology, data analysis, data interpretation, writing-review and editing DC, MB, WM, TN, AW: Data collection, writing-review and editing. 
DD: Study design/methodology, data interpretation, writing-review and editing

YL: Supervision, funding, data collection, data interpretation, writing-review and editing

LM, JS, YL: have verified the underlying data.

All authors read, revised and approved the final manuscript.

\section{Acknowledgements}

Not applicable

\section{References}

1. Lee YC, Bingham CO, 3rd, Edwards RR, Marder W, Phillips K, Bolster MB, et al. Association between pain sensitization and disease activity in patients with rheumatoid arthritis: a cross-sectional study. Arthritis Care Res (Hoboken). 2018;70:197-204.

2. Kosek E, Cohen M, Baron R, Gebhart GF, Mico JA, Rice ASC, et al. Do we need a third mechanistic descriptor for chronic pain states? Pain. 2016 Jul;157:1382-6.

3. Clauw DJ, Crofford LJ. Chronic widespread pain and fibromyalgia: what we know, and what we need to know. Best Pract Res Clin Rheumatol. 2003;17:685-701.

4. Laursen BS, Bajaj P, Olesen AS, Delmar C, Arendt-Nielsen L. Health related quality of life and quantitative pain measurement in females with chronic non-malignant pain. Eur J Pain. 2005;9:267-75.

5. Heisler AC, Song J, Muhammad LN, Wohlfahrt A, Marder W, Bolster MB, et al. Association of dysregulated central pain processing and response to disease-modifying anti-rheumatic drug therapy in rheumatoid arthritis. Arthritis Rheumatol. 2020;72:2017-24.

6. Cruz-Almeida Y, Fillingim RB. Can quantitative sensory testing move us closer to mechanism-based pain management? Pain Med. 2014;15:61-72.

7. Leffler AS, Kosek E, Lerndal T, Nordmark B, Hansson P. Somatosensory perception and function of diffuse noxious inhibitory controls (DNIC) in patients suffering from rheumatoid arthritis. Eur $\mathrm{J}$ Pain. 2002;6:161-76.

8. Edwards RR, Wasan AD, Bingham CO 3rd, Bathon J, Haythornthwaite JA, Smith MT, et al. Enhanced reactivity to pain in patients with rheumatoid arthritis. Arthritis Res Ther. 2009;11:R61.

9. Neville SJ, Clauw AD, Moser SE, Urquhart AG, Clauw DJ, Brummett CM, et al. Association between the 2011 fibromyalgia survey criteria and multisite pain sensitivity in knee osteoarthritis. Clin J Pain. 2018;34:909-17. 
10. Brummett CM, Goesling J, Tsodikov A, Meraj TS, Wasserman RA, Clauw DJ, et al. Prevalence of the fibromyalgia phenotype in patients with spine pain presenting to a tertiary care pain clinic and the potential treatment implications. Arthritis Rheum. 2013;65:3285-92.

11. Aoyagi K, He J, Nicol AL, Clauw DJ, Kluding PM, Jernigan S, et al. A subgroup of chronic low back pain patients with central sensitization. Clin J Pain. 2019;35:869-79.

12. Wolfe F, Clauw DJ, Fitzcharles MA, Goldenberg DL, Häuser W, Katz RS, et al. Fibromyalgia criteria and severity scales for clinical and epidemiological studies: a modification of the ACR Preliminary Diagnostic Criteria for Fibromyalgia. J Rheum. 2011;38:1113-22.

13. Moore RL, Clifford AM, Moloney N, Doody C, Smart KM, O'Leary H. The relationship between clinical and quantitative measures of pain sensitization in knee osteoarthritis. Clin J Pain. 2020;36:336-43.

14. Joharatnam N, McWilliams DF, Wilson D, Wheeler M, Pande I, Walsh DA. A cross-sectional study of pain sensitivity, disease-activity assessment, mental health, and fibromyalgia status in rheumatoid arthritis. Arthritis Res Ther. 2015;17:11.

15. Felson DT, Anderson JJ, Boers M, Bombardier C, Chernoff M, Fried B, et al. The American College of Rheumatology preliminary core set of disease activity measures for rheumatoid arthritis clinical trials. The Committee on Outcome Measures in Rheumatoid Arthritis Clinical Trials. Arthritis Rheum. 1993;36:729-40.

16. England BR, Tiong BK, Bergman MJ, Curtis JR, Kazi S, Mikuls TR, et al. 2019 update of the American College of Rheumatology recommended rheumatoid arthritis disease activity measures. Arthritis Care Res. 2019;71:1540-55.

17. Cicchetti DV. Guidelines, criteria, and rules of thumb for evaluating normed and standardized assessment instruments in psychology. Psychol Assess. 1994;6:284-90.

18. Wolfe F, Clauw DJ, Fitzcharles MA, Goldenberg DL, Häuser W, Katz RL, et al. 2016 revisions to the 2010/2011 fibromyalgia diagnostic criteria. Semin Arthritis Rheum. 2016;46:319-29.

19. Shresher NM, Mohamed AE, Elshahaly MH. Performance of 2016 revised fibromyalgia diagnostic criteria in patients with rheumatoid arthritis. Rheumatol Int. 2019;39:1703-10.

20. Sullivan MJL, Bishop SR, Pivik J. The Pain Catastrophizing Scale: development and validation. Psychol Assess. 1995; 7:524-32.

21. Geisser ME, Gracely RH, Giesecke T, Petzke FW, Williams DA, Clauw DJ. The association between experimental and clinical pain measures among persons with fibromyalgia and chronic fatigue syndrome. Eur J Pain. 2007;11:202-7.

22. Melzack R. The short-form McGill Pain Questionnaire. Pain. 1987 Aug;30(2):191-197. 
23. Neogi T, Frey-Law L, Scholz J, Niu J, Arendt-Nielsen L, Woolf C, et al. Sensitivity and sensitisation in relation to pain severity in knee osteoarthritis: trait or state? Ann Rheum Dis. 2015 Apr;74(4):682-8.

24. Riley JL 3rd, Robinson ME, Wise EA, Myers CD, Fillingim RB. Sex differences in the perception of noxious experimental stimuli: a meta-analysis. Pain. 1998;74:181-87.

25. Lee YC, Nassikas NJ, Clauw DJ. The role of the central nervous system in the generation and maintenance of chronic pain in rheumatoid arthritis, osteoarthritis and fibromyalgia. Arthritis Res Ther. 2011;28;13:211.

26. Wolfe F, Michaud K. Severe rheumatoid arthritis (RA), worse outcomes, comorbid illness, and sociodemographic disadvantage characterize RA patients with fibromyalgia. J Rheumatology. 2004;31:695-700.

27. Duffield SJ, Miller N, Zhao S, Goodson NJ. Concomitant fibromyalgia complicating chronic inflammatory arthritis: a systematic review and meta-analysis. Rheumatology (Oxford). 2018;57:1453-60.

28. LeResche L, Turner JA, Saunders K, Shortreed SM, Von Korff M. Psychophysical tests as predictors of back pain chronicity in primary care. J Pain. 2013;14:1663-70.

29. Müller M, Limacher A, Agten CA, Treichel F, Heini P, Seidel U, Andersen OK, Arendt-Nielsen L, Jüni P, Curatolo M. Can quantitative sensory tests predict failed back surgery?: A prospective cohort study. Eur J Anaesthesiol. 2019;36:695-704.

30. Schweinhardt P, Kalk N, Wartolowska K, Chessell I, Wordsworth P, Tracey I. Investigation into the neural correlates of emotional augmentation of clinical pain. Neuroimage. 2008;40:759-66.

31. Napadow V, LaCount L, Park K, As-Sanie S, Clauw DJ, Harris RE. Intrinsic brain connectivity in fibromyalgia is associated with chronic pain intensity. Arthritis Rheum. 2010;62:2545-55.

\section{Tables}

Table 1: Baseline characteristics $(\mathrm{N}=285)$ * 


\begin{tabular}{|ll|}
\hline Variable & Mean (SD) or \% \\
\hline Age (years) & $54.7(13.74)$ \\
\hline Female & $82.1 \%$ \\
\hline Caucasian & $74.7 \%$ \\
\hline BMI (kg/m ${ }^{2}$ ) & $28.58(6.62)$ \\
\hline Seropositive, $\%$ & $78.3 \%$ \\
\hline Site, \% of enrolled & \\
\hline Brigham/MGH & $51.9 \%$ \\
\hline Boston University & $10.2 \%$ \\
\hline Michigan University & $19.3 \%$ \\
\hline Johns Hopkins & $18.6 \%$ \\
\hline CDAl & $24.56(14.25)$ \\
\hline Patient global & $4.23(2.44)$ \\
\hline Physician global & $3.68(2.28)$ \\
\hline Swollen joint count & $5.26(5.25)$ \\
\hline Tender joint count & $10.89(8.6)$ \\
\hline CRP (mg/L) & $8.15(12.45)$ \\
\hline FSQ score & $11.22(6.08)$ \\
\hline WPI score & $5.95(4.32)$ \\
\hline SSS score & $5.27(2.65)$ \\
\hline QST & $12.54(14.63)$ \\
\hline Thumbnail PPT (kgf) & $3.67(1.95)$ \\
\hline Trapezius PPT (kgf) & $2.93(1.65)$ \\
\hline Wrist PPT (kgf) & $2.93(1.59)$ \\
\hline Knee PPT (kgf) & $5.41(2.84)$ \\
\hline Wrist TS & $13.06(14.78)$ \\
\hline Arm TS & \\
\hline CPM & \\
\hline
\end{tabular}


${ }^{*}$ CDAl $n=243$; patient global $n=243$; thumbnail PPT, trapezius PPT, wrist PPT $n=284$; knee PPT $n=283$; wrist TS n=282; arm TS n=281; CPM n=279.

BMI body mass index; MGH Massachusetts General Hospital, CDAl clinical disease activity index; CRP Creactive protein; FSQ fibromyalgia survey questionnaire; WPI widespread pain index; SSS symptom severity score; QST quantitative sensory index; PPT pressure pain threshold; TS temporal summation; CPM conditioned pain modulation

Table 2: Unadjusted correlations between QST measures and FSQ after stratification by sex

\begin{tabular}{lll}
\hline QST & Men & Women \\
& $\begin{array}{l}\text { Correlation Coefficient } \\
\mathbf{( 9 5 \% ~ C o n f i d e n c e ~ L i m i t s ) ~}\end{array}$ & $\begin{array}{l}\text { Correlation Coefficient } \\
\mathbf{( 9 5 \% ~ C o n f i d e n c e ~ L i m i t s ) ~}\end{array}$ \\
\hline PPT thumb & $-0.15(-0.41,0.13)$ & $-0.22(-0.34,-0.09)$ \\
\hline PPT trapezius & $-0.36(-0.57,-0.09)$ & $-0.20(-0.32,-0.08)$ \\
\hline PPT wrist & $-0.23(-0.48,0.05)$ & $-0.26(-0.38,-0.14)$ \\
\hline PPT knee & $-0.31(-0.54,-0.03)$ & $-0.30(-0.41,-0.18)$ \\
\hline TS wrist & $0.08(-0.20,0.35)$ & $0.15(0.02,0.27)$ \\
\hline TS arm & $0.07(-0.21,0.34)$ & $0.14(0.01,0.27)$ \\
\hline CPM & $0.00(-0.28,0.28)$ & $-0.01(-0.14,0.12)$
\end{tabular}

QST quantitative sensory testing; PPT pressure pain threshold; TS temporal summation; CPM conditioned pain modulation

Table 3: Unadjusted correlations between QST measures and the individual components of FSQ, the WPI and SSS 


\begin{tabular}{lll} 
& WPI & SSS \\
\hline QST & $\begin{array}{l}\text { Correlation Coefficient (95\% Confidence } \\
\text { Limits) }\end{array}$ & $\begin{array}{l}\text { Correlation Coefficient (95\% Confidence } \\
\text { Limits) }\end{array}$ \\
\hline PPT thumb & $-0.13(-0.24,-0.01)$ & $-0.26(-0.36,-0.14)$ \\
\hline $\begin{array}{l}\text { PPT } \\
\text { trapezius }\end{array}$ & $-0.18(-0.29,-0.07)$ & $-0.25(-0.36,-0.14)$ \\
\hline PPT wrist & $-0.20(-0.31,-0.08)$ & $-0.29(-0.39,-0.18)$ \\
\hline PPT knee & $-0.23(-0.34,-0.12)$ & $-0.31(-0.41,-0.20)$ \\
\hline TS wrist & $0.12(0.00,0.23)$ & $0.16(0.04,0.27)$ \\
\hline TS arm & $0.10(-0.02,0.22)$ & $0.13(0.01,0.24)$ \\
\hline CPM & $0.01(-0.11,0.13)$ & $0.01(-0.11,0.13)$
\end{tabular}

QST quantitative sensory testing; PPT pressure pain threshold; TS temporal summation; CPM conditioned pain modulation

\section{Figures}


Spearman Correlations of QST with FSQ

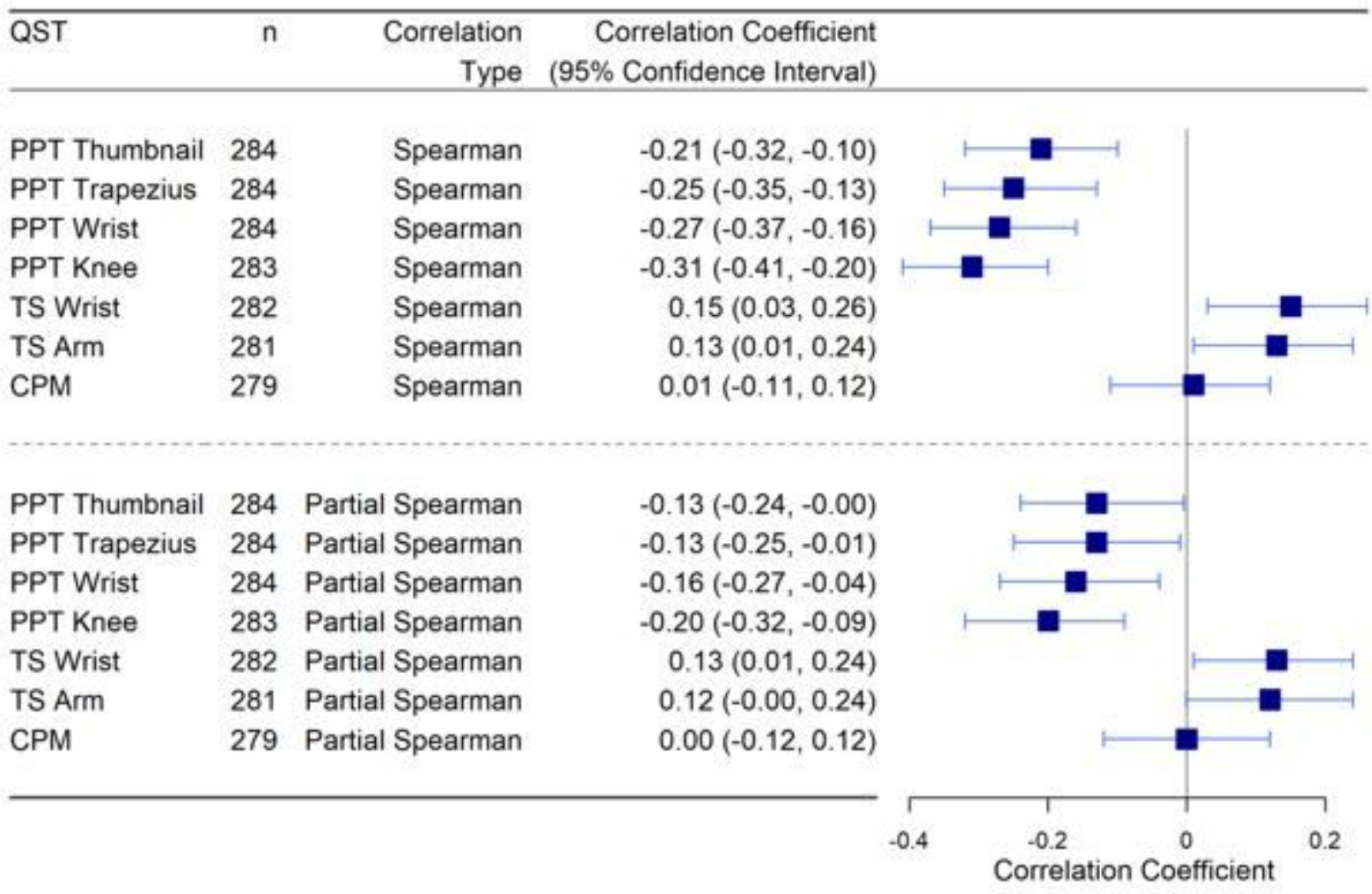

Adjusted for age, sex, race, BMI, seropositivity, swollen joint count, CRP, pain catastrophizing, and site.

QST quantitative sensory testing; FSQ fibromyalgia survey questionnaire; PPT pressure pain threshold; TS temporal summation; CPM conditioned pain modulation

\section{Figure 1}

Unadjusted and adjusted correlations between QST measures and FSQ 